\title{
Associations of platelet-activating factor acetylhydrolase gene polymorphisms with risk of ischemic stroke
}

\author{
YONGSHENG MA
}

\author{
Department of Geriatrics, Weifang People's Hospital, Weifang, Shandong 261041, P.R. China
}

Received July 23, 2015; Accepted December 11, 2015

DOI: $10.3892 /$ br. 2015.560

\begin{abstract}
Platelet-activating factor acetylhydrolase (PAF-AH) has an important function in the pathogenesis of ischemic stroke. The aim of the present study was to investigate the correlation between the variation of polymorphisms $(\mathrm{R} 92 \mathrm{H}$ and V279F) in PAF-AH and ischemic stroke. A total of 375 patients with ischemic stroke and 370 healthy controls were recruited into the study. Polymorphisms of V279F and $\mathrm{R} 92 \mathrm{H}$ in PAF-AH were detected by polymerase chain reaction and DNA direct sequencing method. No significant association was observed between V279F and ischemic stroke. However, the $\mathrm{RH}+\mathrm{HH}$ genotype, $\mathrm{RH}$ genotype and $\mathrm{H}$ allele of $\mathrm{R} 92 \mathrm{H}$ were significantly associated with an increased risk of ischemic stroke $(\mathrm{P}=0.02, \mathrm{P}=0.03$ and $\mathrm{P}=0.02$, respectively), In addition, these correlations remained following adjustment for confounding risk factors of stroke. Furthermore, subgroup analysis showed that a significant association with $\mathrm{R} 92 \mathrm{H}$ was identified in the large-artery atherosclerotic stroke subgroup. These findings indicated that variation of $\mathrm{R} 92 \mathrm{H}$ in the PAF-AH gene may contribute to ischemic stroke susceptibility in the population studied.
\end{abstract}

\section{Introduction}

Stroke is the second cause of fatality and the leading cause of disability worldwide. Currently, in developing countries its prevalence is also increasing rapidly. In China, stroke is a major cause of fatality (1). Presently, ischemic stroke is the most common type of stroke in China (2). Numerous independent and major risk factors, such as hypertension, diabetes mellitus, hypercholesterolemia, smoking, alcohol consumption, family history, lack of exercise, diet and lifestyle, are known causes of ischemic stroke (3).

Previous studies have proved that the heritable elements can influence the pathogenesis of ischemic stroke. A significant

Correspondence to: Dr Yongsheng Ma, Department of Geriatrics, Weifang People's Hospital, 151 Guangwen Street, Weifang, Shandong 261041, P.R. China

E-mail: mys2004097@163.com

Key words: polymorphisms, ischemic stroke, platelet-activating factor acetylhydrolase number of genomic alleles and variants have been discovered to increase the risk of ischemic stroke (4); however, these only explain a minor part of the assumed heritability. Exploring the genetic polymorphisms contributing to the susceptibility of ischemic stroke is important. Recent studies have shown that inflammation is one of the key risk factors and has an important role in the development of ischemic stroke (5). Therefore, genes involved in inflammatory responses are under investigation to identify the variants predisposing to ischemic stroke.

Platelet-activating factor acetylhydrolase (PAF-AH), also known as lipoprotein-associated phospholipase A2 (Lp-PLA2), is a newly identified inflammatory enzyme involved in lipoprotein metabolism and inflammatory pathways (6). PAF-AH may have an important role in the pathophysiology of inflammation. Clinical and epidemiological studies have indicated that elevated PAF-AH concentrations are associated with incident and recurrent stroke events, as this enzyme exhibits proinflammatory and oxidative activities (7). Several other studies have examined the association of PAF-AH gene polymorphisms with coronary artery disease. V279F in exon 9 of the PAF-AH gene is associated with coronary artery disease and carotid atherosclerosis in the Japanese population. $\mathrm{R} 92 \mathrm{H}$ in exon 4 is associated with coronary artery disease in the USA (8). According to this information, we hypothesized that the genetic variants in PAF-AH may have a critical role in the susceptibility to ischemic stroke. To confirm this hypothesis, a case-control study was conducted to investigate the association between two PAF-AH polymorphisms (V279F and R92H) with ischemic stroke in an eastern Chinese population.

\section{Materials and methods}

Study population. The present study was a hospital-based, case-control study. A total of 375 patients with ischemic stroke and 370 healthy controls were diagnosed at Weifang People's Hospital (Weifang, Shandong, China) were recruited between January 2011 and February 2015. The ischemic stroke diagnoses were carried out according to the World Health Organization guidelines (9) and the reported procedures. According to the TOAST classification, ischemic stroke can be divided into five subtypes: i) Large-artery atherosclerosis (LAA), ii) small-vessel occlusion (SVO), iii) cardioembolism (CE), iv) stroke of other determined etiology, and v) stroke of undetermined etiology (10). Patients with LAA and SVO, two of the most common subtypes of ischemic stroke, were 
included, while the other subtypes were excluded. All the subjects in the control group were free of clinical or radiological evidence of stroke and other neurological diseases. Those having mental or significant physical diseases, as well as familial or self-psychiatric history, were excluded.

The completion of questionnaires for each subject was performed by trained interviewers, and their clinical assay markers were measured using standard laboratory procedures. The study was approved by the Ethics Committee of the Weifang People's Hospital and written informed consent was obtained from every recruited subject. This study was conducted in accordance with the Declaration of Helsinki.

DNA extraction and genotyping. Genomic DNA extraction was executed using commercial kits designed for extracting blood DNA (Qiagen, Valencia, CA, USA) and following the manufacturer's protocol. TE buffer was used to dissolve the extracted DNA. The resulted DNA solution was stored at $-20^{\circ} \mathrm{C}$ until further use, and was also used as a template for the following polymerase chain reaction (PCR). The sequences of the primers for PCR are shown in Table I. PCR amplification was performed in a total volume of $10 \mu \mathrm{l}$ that contained $1 \mathrm{X} \mathrm{GC}$ buffer I (Takara, Otsu, Japan), $3.0 \mathrm{mM} \mathrm{Mg}^{2+}$ (Takara), $0.3 \mathrm{mM}$ deoxyribonucleotide triphosphate (dNTP) (Generay Biotech, Shanghai, China), 1 unit HotStarTaq polymerase (Qiagen, Hilden, Germany), $1 \mu$ l each primer (Sangon, Shanghai, China) and $1 \mu \mathrm{l}$ genomic DNA. The PCR cycling program was set at $95^{\circ} \mathrm{C}$ for $2 \mathrm{~min}$, followed by 11 cycles of $94^{\circ} \mathrm{C}$ for $20 \mathrm{sec}, 65^{\circ} \mathrm{C}$ (decreased $0.5^{\circ} \mathrm{C}$ per cycle) for $40 \mathrm{sec}, 72^{\circ} \mathrm{C}$ for $1.5 \mathrm{~min}$, and subsequently 24 cycles of $94^{\circ} \mathrm{C}$ for $20 \mathrm{sec}, 59^{\circ} \mathrm{C}$ for $30 \mathrm{sec}$, and $72^{\circ} \mathrm{C}$ for $1.5 \mathrm{~min}$, and a final extension at $72^{\circ} \mathrm{C}$ for $2 \mathrm{~min}$. Multiplex PCR products were checked for quality and yield by running $5 \mu \mathrm{l}$ in $2 \%$ agarose-TBE gels. PCR products $(15 \mu \mathrm{l})$ were treated with 5 units of shrimp alkaline phosphatase and 2 units of exonuclease I to remove excess dNTPs and primers, respectively. The results of electrophoresis are shown in Figs. 1 and 2.

Statistical analysis. Continuous variables are presented as mean \pm standard deviation and categorical variables as percentages. Continuous variables were compared using student's t-test. Categorical variables were compared by $\chi^{2}$ test. Differences of the distributions of alleles and genotypes between cases and controls were analyzed using $\chi^{2}$ test. All the genotype frequencies were checked for Hardy-Weinberg analysis in two groups with the $\chi^{2}$ test.

The association of the PAF-AH gene polymorphisms with ischemic stroke was evaluated by computing the odds ratios (OR) and 95\% confidence intervals (CI) from logistic regression analyses following adjustment for confounding risk factors. $\mathrm{P}<0.05$ was considered to indicate a statistically significant difference for all statistical analyses. All the statistical analyses were carried out with Stata 12.0 software (StataCorp, College Station, TX, USA).

\section{Results}

Characteristics. The clinical characteristics of the control subjects and ischemic stroke patients are shown in Table II. There were no significant differences in age, gender or body
Table I. Primer sequences of V279F and R92H.

\begin{tabular}{ll}
\hline SNPs & \multicolumn{1}{c}{ Primer sequences } \\
\hline R92H & $\begin{array}{l}\text { Forward: 5' CAATCACCACAGCAGCCTAA3' } \\
\text { Reverse: 5' TCCCATCCAACTCAGAATGG3' }\end{array}$ \\
V279F & Forward: 5' TTTATGGGGGCAAAAGAATAGCC3' \\
& Reverse: 5' AACCATCCCCATGAAATSAACAAT3'
\end{tabular}

SNP, single nucleotide polymorphism.

Table II. Clinical characteristics of the study subjects.

\begin{tabular}{lcrr}
\hline Characteristics & Cases & Control & P-value \\
\hline Age $^{\mathrm{a}}$, years & $63.4 \pm 4.73$ & $60.1 \pm 8.09$ & 0.81 \\
Males, n (\%) & $254(67.7)$ & $243(65.7)$ & 0.74 \\
BMI $^{\mathrm{a}}, \mathrm{kg} / \mathrm{m}^{2}$ & $27.2 \pm 2.91$ & $26.8 \pm 2.03$ & 0.23 \\
Hypertension, n (\%) & $263(70.1)$ & $218(58.9)$ & $<0.01$ \\
Diabetes, n (\%) & $131(34.9)$ & $69(18.6)$ & $<0.01$ \\
Hyperlipidemia, n (\%) & $167(44.5)$ & $129(34.9)$ & $<0.01$ \\
Smoking, n (\%) & $131(34.9)$ & $67(18.1)$ & $<0.01$ \\
\hline
\end{tabular}

${ }^{\text {a }}$ Data are mean \pm standard deviation. BMI, body mass index.

mass index. The genotype and allele frequencies of the two PAF-AH polymorphisms in patients and control subjects are shown in Tables III and IV. All the genotype distributions in the patients and controls were in the Hardy-Weinberg equilibrium. As shown in Tables III and IV, there was no significant difference in the distributions of genotypes and alleles of V279F between ischemic stroke patients and controls. By contrast, the significant association was observed for $\mathrm{R} 92 \mathrm{H}$ in a dominant model. A higher frequency of the $\mathrm{HH}+\mathrm{RH}$ genotype for $\mathrm{R} 92 \mathrm{H}$ was observed in all the participants with ischemic stroke $(\mathrm{OR}=1.44 ; 95 \% \mathrm{CI}, 1.06-2.01 ; \mathrm{P}=0.02)$. The $\mathrm{RH}$ genotype (28.8\%) of $\mathrm{R} 92 \mathrm{H}$ was represented at an increased frequency in the group of patients $(\mathrm{OR}=1.42 ; 95 \% \mathrm{CI}, 1.02-1.97 ; \mathrm{P}=0.03)$. The frequency of $\mathrm{R} 92 \mathrm{H} \mathrm{H}$ allele was significantly higher in patients with ischemic stroke compared to the control group $(\mathrm{OR}=1.41 ; 95 \% \mathrm{CI}, 1.06-1.73 ; \mathrm{P}=0.02)$.

Association between polymorphisms and ischemic stroke. The association between the polymorphisms and the disease was assessed using univariate or multivariate logistic regression analyses. Logistic regression analyses revealed that following adjustment for the confounding factors (hypertension, diabetes mellitus, hyperlipidemia and smoking), the presence of the $\mathrm{RH}+\mathrm{HH}$ genotype and the $\mathrm{RH}$ genotype of single nucleotide polymorphism $\mathrm{R} 92 \mathrm{H}$ was associated with a higher risk of ischemic stroke $(\mathrm{OR}=1.42 ; 95 \% \mathrm{CI}, 1.02-2.00 ; \mathrm{P}=0.04$; and $\mathrm{OR}=1.41 ; 95 \% \mathrm{CI}, 1.01-2.08 ; \mathrm{P}=0.04$, respectively).

The statistical differences between the PAF-AH gene and different subtypes of ischemic stroke were analyzed. The $\mathrm{HH}+\mathrm{RH}$ genotype and the RH genotype of the R92H gene were significantly associated with large-artery atherosclerotic 
Table III. Genotype and allele distributions of R92H in patients with ischemic stroke and the controls.

\begin{tabular}{lccccc}
\hline R92H & Cases, $(\%)$ & Control, $(\%)$ & OR $(95 \% \mathrm{CI})$ & P-value & Adjusted OR (95\% CI) \\
\hline Genotype & & & & \\
RR & $259(69.1)$ & $287(77.6)$ & Reference & Reference & \\
RH & $104(27.7)$ & $79(21.4)$ & $1.42(1.02-1.97)$ & 0.03 & $1.41(1.01-2.08)$ \\
HH & $12(3.2)$ & $4(1.1)$ & $2.12(0.62-7.41)$ & 0.19 & $1.72(0.48-6.15)$ \\
(HH+RH)/RR & $116 / 259$ & $83 / 287$ & $1.44(1.06-2.01)$ & 0.02 & $1.42(1.02-2.00)$ \\
HH/(RH+RR) & $12 / 363$ & $4 / 386$ & $2.11(0.54-6.39)$ & 0.25 & $1.55(0.44-5.59)$ \\
Allele & & & & & 0.04 \\
R & $622(82.9)$ & $653(87.8)$ & Reference & & 0.48 \\
H & $128(17.1)$ & $87(12.2)$ & $1.41(1.06-1.73)$ & 0.02 & \\
\hline
\end{tabular}

OR, odds ratio; CI, confidence interval.

Table IV. Genotype and allele distributions of V279F in patients with ischemic stroke and the controls.

\begin{tabular}{|c|c|c|c|c|c|c|}
\hline V279F & Cases, n (\%) & Control, n (\%) & OR $(95 \% \mathrm{CI})$ & P-value & Adjusted OR (95\% CI) & P-value \\
\hline \multicolumn{7}{|l|}{ Genotype } \\
\hline VV & $338(90.1)$ & $334(90.2)$ & Reference & Reference & & \\
\hline VF & $35(9.3)$ & $33(8.9)$ & $0.93(0.57-1.46)$ & 0.77 & $1.02(0.63-1.62)$ & 0.88 \\
\hline $\mathrm{FF}$ & $2(0.5)$ & $3(0.8)$ & $0.23(0.02-2.12)$ & 0.35 & $0.21(0.02-2.01)$ & 0.17 \\
\hline$(\mathrm{FF}+\mathrm{VF}) / \mathrm{VV}$ & $37 / 338$ & $36 / 334$ & $0.86(0.54-1.27)$ & 0.54 & $0.92(0.61-1.51)$ & 0.83 \\
\hline $\mathrm{FF} /(\mathrm{VF}+\mathrm{VV})$ & $2 / 373$ & $3 / 367$ & $0.24(0.02-2.22)$ & 0.36 & $0.21(0.02-2.10)$ & 0.18 \\
\hline \multicolumn{7}{|l|}{ Allele } \\
\hline $\mathrm{V}$ & $711(94.8)$ & $701(94.7)$ & Reference & & & \\
\hline $\mathrm{F}$ & $39(5.2)$ & $39(5.3)$ & $0.82(0.44-1.24)$ & 0.35 & & \\
\hline
\end{tabular}

OR, odds ratio; CI, confidence interval.

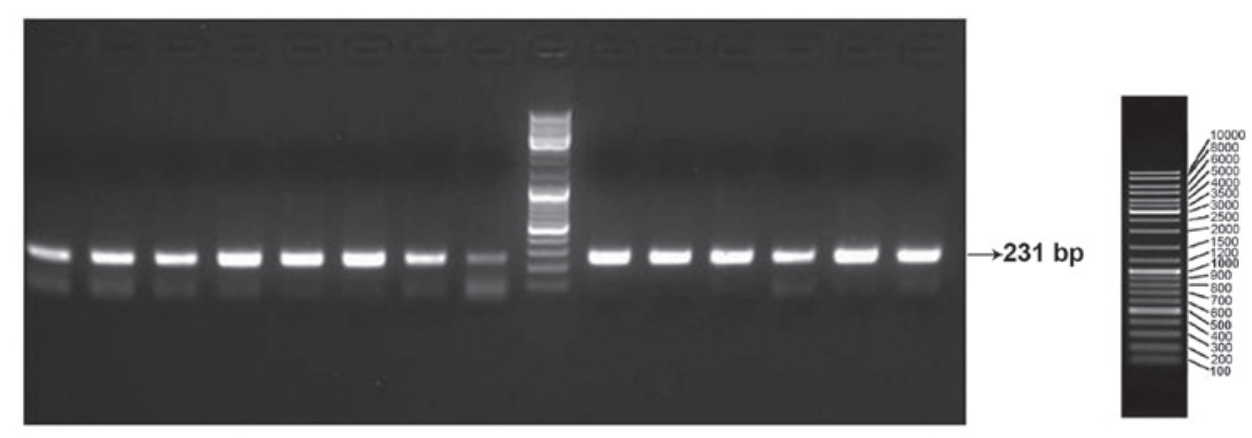

Figure 1. Electrophoresis of R92H.

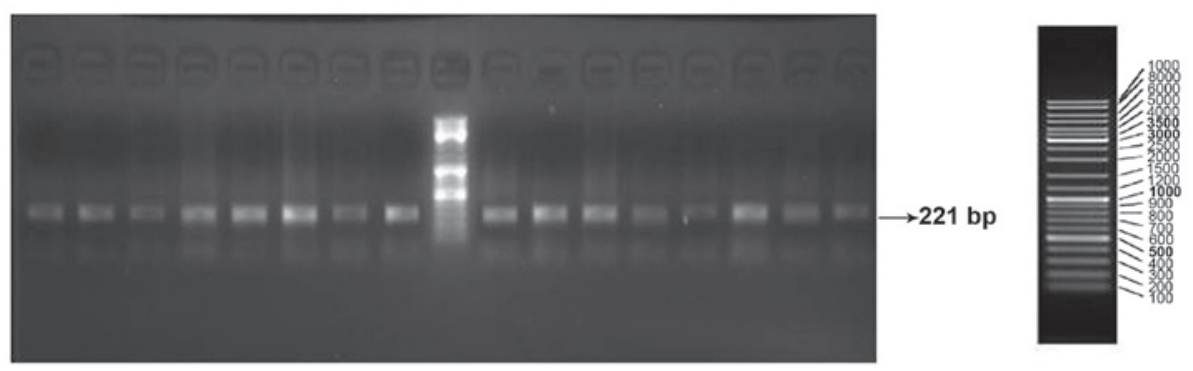

Figure 2. Electrophoresis of V279F. 
Table V. Genotype and allele distributions of R92H and its associations with the stroke subtypes.

\begin{tabular}{|c|c|c|c|c|c|c|}
\hline $\mathrm{R} 92 \mathrm{H}$ & Cases, n (\%) & Control, n (\%) & OR $(95 \% \mathrm{CI})$ & P-value & Adjusted OR (95\% CI) & P-value \\
\hline \multicolumn{7}{|c|}{ LAA subgroup } \\
\hline $\mathrm{RR}$ & $133(64.4)$ & $151(73.7)$ & Reference & & & \\
\hline RH & $70(32.4)$ & $42(20.5)$ & $1.73(1.19-2.52)$ & $<0.01$ & $1.67(1.13-2.48)$ & 0.01 \\
\hline $\mathrm{HH}$ & $6(3.2)$ & $12(5.9)$ & $3.67(1.06-12.76)$ & 0.06 & $3.02(0.81-11.27)$ & 0.10 \\
\hline \multicolumn{7}{|c|}{ Dominant model } \\
\hline $\mathrm{RR}$ & $133(64.4)$ & $151(73.7)$ & Reference & & & \\
\hline $\mathrm{HH}+\mathrm{RH}$ & $76(36.4)$ & $54(26.3)$ & $1.82(1.27-2.62)$ & $<0.01$ & $1.73(1.18-2.55)$ & $<0.01$ \\
\hline \multicolumn{7}{|c|}{ Recessive model } \\
\hline $\mathrm{RR}+\mathrm{RH}$ & 203 (97.1) & $193(94.1)$ & Reference & & & \\
\hline $\mathrm{HH}$ & $6(2.9)$ & $12(11.4)$ & $0.75(0.21-1.90)$ & 0.11 & $0.63(0.14-1.72)$ & 0.15 \\
\hline \multicolumn{7}{|l|}{ Allele } \\
\hline $\mathrm{R}$ & $336(80.4)$ & $344(83.9)$ & Reference & & & \\
\hline $\mathrm{H}$ & $82(19.6)$ & $66(16.1)$ & $1.74(1.26-2.39)$ & 0.23 & & \\
\hline \multicolumn{7}{|c|}{ SVO subgroup } \\
\hline $\mathrm{RR}$ & $126(75.9)$ & $106(64.2)$ & Reference & & & \\
\hline RH & $39(23.5)$ & 56 (33.9) & $1.09(0.71-1.68)$ & 0.69 & $1.11(0.71-1.73)$ & 0.65 \\
\hline $\mathrm{HH}$ & $1(0.6)$ & $3(1.8)$ & $0.59(0.07-5.31)$ & 1.00 & $0.33(0.03-3.36)$ & 0.35 \\
\hline \multicolumn{7}{|c|}{ Dominant model } \\
\hline $\mathrm{RR}$ & $126(75.9)$ & $106(64.2)$ & Reference & & & \\
\hline $\mathrm{HH}+\mathrm{RH}$ & $40(24.1)$ & $59(35.8)$ & $1.07(0.70-1.64)$ & 0.75 & $1.07(0.69-1.66)$ & 0.78 \\
\hline \multicolumn{7}{|c|}{ Recessive model } \\
\hline RR+RH & 165 (99.4) & $162(98.2)$ & Reference & & & \\
\hline $\mathrm{HH}$ & $1(0.6)$ & $3(1.8)$ & $0.58(0.06-5.19)$ & 0.99 & $0.32(0.03-3.23)$ & 0.33 \\
\hline \multicolumn{7}{|l|}{ Allele } \\
\hline $\mathrm{R}$ & $291(87.7)$ & $268(81.2)$ & Reference & & & \\
\hline $\mathrm{H}$ & $41(12.3)$ & $62(18.7)$ & $1.04(0.70-1.53)$ & 0.85 & & \\
\hline
\end{tabular}

OR, odds ratio; CI, confidence interval.

stroke, even after adjusting for the confounding factors $(\mathrm{OR}=1.73 ; 95 \% \mathrm{CI}, 1.18-2.55 ; \mathrm{P}<0.01 ;$ and $\mathrm{OR}=1.67$; 95\% CI, 1.13-2.48; $\mathrm{P}=0.01$, respectively). However, the same differences were not observed between the remaining small vessel occlusive stroke subgroup and all genetic models $(\mathrm{P}>0.05)$, as shown in Table V.

\section{Discussion}

The present study investigated the association between the PAF-AH genotypes and the risk of ischemic stroke in the eastern Chinese Han population. The major finding of this study is that in the Chinese Han population the frequencies of the minor alleles of the $\mathrm{R} 92 \mathrm{H}$ polymorphisms of the PAF-AH gene were significantly higher in ischemic stroke patients. This difference remained following all the factor-related adjustments. However, no association of V279F with the risk of ischemic stroke was identified.

Several previous studies have explored the association between the PAF-AH polymorphism and cardiovascular disease. However, the results of these studies were inconsistent. Li et al (11) reported that the V279F variant was associated with coronary artery disease in the Chinese Han population. However, the association between the V279F variant and cardiovascular disease was not found in the South Korean population (12). With regards to ischemic stroke, Hiramoto et al (13) identified an association with the $\mathrm{FF}+\mathrm{VF}$ genotype and the F allele. In the present study, this association has not been replicated in the population of eastern China. Two points should be considered with regards to these findings. Genetic heterogeneity may be an important factor. Firstly, genotype frequencies of the V279F in the control subjects $(88 \% \mathrm{VV}, 11 \% \mathrm{VF}$, and $1 \% \mathrm{FF})$ differ from those in the Japanese populations (75\% VV, 22\% VF, and 3\% FF). Secondly, the study by Hiramoto et al (13) was conducted with a relatively small sample size and there was a low frequency of homozygosity of the minor allele. It may be more likely to produce false positive results.

The $\mathrm{R} 92 \mathrm{H}$ is a polymorphism, with a non-synonymous change located within the coding region of the Lp-pla2 gene in exon 4, which should result in an arginine-to-histidine substitution at position 92. The association of R92H with cardiovascular disease is also contradictory. Sutton et al (8) reported that the $\mathrm{R} 92 \mathrm{H}$ polymorphism was significantly 
associated with the risk of CHD, with the $\mathrm{R} 92 \mathrm{H}$ variant allele observed more frequently in the experiment group compared to the control group. Zheng et al (14) identified the significant association of the $\mathrm{R} 92 \mathrm{H}$ variant with premature myocardial infarction in the Chinese population. In the present study, the focus was on the association between $\mathrm{R} 92 \mathrm{H}$ in the PAF-AH gene and ischemic stroke. The $\mathrm{HH}+\mathrm{RH}$ genotype, the $\mathrm{RH}$ genotype and the $\mathrm{H}$ allele of $\mathrm{R} 92 \mathrm{H}$ were significantly associated with the increased risk of ischemic stroke in the Chinese Han population.

The association between $\mathrm{R} 92 \mathrm{H}$ in the PAF-AH gene and ischemic stroke in the Chinese population can possibly be explained as follows: i) Several previous studies have investigated the association between the PAF-AH polymorphism and the risk of ischemic stroke. A study of older individuals by Rosso et al (15) found that PAF-AH was associated with ischemic and hemorrhagic strokes. In the Bruneck study (16), PAF-AH activity was identified as a top hit associated with ischemic stroke. ii) A few studies have investigated the association between the R92H polymorphism and plasma PAF-AH activity. A meta-analysis including a total of 14 studies showed that the $\mathrm{R} 92 \mathrm{H}$ variant shows the strongest association with PAF-AH activity among the variants in PAF-AH gene (17).

When evaluating the association of $\mathrm{R} 92 \mathrm{H}$ with stroke subtypes, a significant association was found for this polymorphism with the LAA subgroup, but not the SVO subgroup. This can be explained by the role of PAF-AH in the process of atherosclerosis. Lyso-PC, the important bioproduct of $\mathrm{PAF}-\mathrm{AH}$, is involved in the inflammatory cytokine production and in the induction of the expression of adhesion molecules and cytokines. It has a chemoattractant property for macrophages, and it induces vascular smooth muscle migration (18). Additionally, Lyso-PC can upregulate PAF-AH activity, resulting in a vicious cycle in which pro-inflammatory mediators are upregulated, contributing to plaque progression and destabilization (19). In addition, oxNEFAs, another detrimental substrate of PAF-AH, can promote atherosclerosis by increasing oxidative stress and the presence of oxidized LDL and other lipoproteins in the plasma and arterial walls, thereby initiating fatty streak formation (20).

There are several limitations in the present study that require discussion, such as the relatively small sample size and the lack of PAF-AH activity measurements. Therefore, further studies including functional evaluations are warranted to elucidate the potential mechanism of these polymorphisms in ischemic stroke.

In conclusion, the present study demonstrated that the PAF-AH gene polymorphism R92H may modify the risk of ischemic stroke in the eastern Chinese Han population. Further studies involving functional evaluations are warranted to clarify the potential mechanism of these polymorphisms in ischemic stroke.

\section{References}

1. Liu L, Wang D, Wong KS and Wang Y: Stroke and stroke care in China: Huge burden, significant workload and a national priority. Stroke 42: 3651-3654, 2011.
2. Thrift AG, Dewey HM, Macdonell RA, McNeil JJ and Donnan GA: Incidence of the major stroke subtypes: Initial findings from the North East Melbourne stroke incidence study (NEMESIS). Stroke 32: 1732-1738, 2001.

3. Flossmann E, Schulz UG and Rothwell PM: Systematic review of methods and results of studies of the genetic epidemiology of ischemic stroke. Stroke 35: 212-227, 2004.

4. Schunkert H, König IR, Kathiresan S, Reilly MP, Assimes TL, Holm H, Preuss M, Stewart AF, Barbalic M, Gieger C, et al: Large-scaleassociation analyses identifies 13 new susceptibility loci for coronary artery disease. Nat Genet 43: 333-338, 2011.

5. Libby P: Inflammation in atherosclerosis. Nature 420: 868-874, 2002.

6. Dada N, Kim NW and Wolfert RL: Lp-PLA2: An emerging biomarker of coronary heart disease. Expert Rev Mol Diagn 2: 17-22, 2002.

7. Shi Y, Zhang P, Zhang L, Osman H, Mohler ER III, Macphee C, Zalewski A, Postle A and Wilensky RL: Role of lipoprotein-associated phospholipase A2 in leukocyte activation and inflammatory responses. Atherosclerosis 191: 54-62, 2007.

8. Sutton BS, Crosslin DR, Shah SH, Nelson SC, Bassil A, Hale AB, Haynes C, Goldschmidt-Clermont PJ, Vance JM, Seo D, et al: Comprehensive genetic analysis of the platelet activating factor acetylhydrolase (PLA2G7) gene and cardiovascular disease in case-control and family datasets. Hum Mol Genet 17: 1318-1328, 2008.

9. Ragoschke-Schumm A, Yilmaz U, Kostopoulos P, et al: 'Stroke room': Diagnosis and treatment as a single location for rapid intraarterial stroke treatment. Cerebrovasc Dis 40: 251-257.

10. Adams HP Jr, Bendixen BH, Kappelle LJ, Biller J, Love BB, Gordon DL and Marsh EE III: Classification of subtype of acute ischemic stroke. Definitions for use in a multicenter clinical trial. TOAST. Trial of Org 10172 in acute stroke treatment. Stroke 24: 35-41, 1993.

11. Li L, Qi L, Lv N, Gao Q, Cheng Y, Wei Y, Ye J, Yan X and Dang A: Association between lipoprotein-associated phospholipase A2 gene polymorphism and coronary artery disease in the Chinese Han population. Ann Hum Genet 75: 605-611, 2011.

12. Jang Y, Kim OY, Koh SJ, Chae JS, Ko YG, Kim JY, Cho H, Jeong TS, Lee WS, Ordovas JM and Lee JH: The Val279Phe variant of the lipoprotein-associated phospholipase A2 gene is associated with catalytic activities and cardiovascular disease in Koreanmen. J Clin Endocrinol Metab 91: 3521-3527, 2006.

13. Hiramoto M, Yoshida H, Imaizumi T, Yoshimizu N and Satoh K: A mutation in plasma platelet-activating factor acetylhydrolase (Val279->Phe) is a genetic risk factor for stroke. Stroke 28: 2417-2420, 1997

14. Zheng GH, Xiong SQ, Chen HY, Mei LJ and Wang T: Associations of platelet-activating factor acetylhydrolase (PAFAH) gene polymorphisms with circulating PAF-AH levels and risk of coronary heart diseaseor blood stasis syndrome in the Chinese Han population. Mol Biol Rep 41: 7141-7151, 2014.

15. Rosso C, Rosenbaum D, Pires C, Cherfils C, Koujah N, Mestari F, Gillet E, Crozier S, Sahli-Amor M, Samson Y, et al: Lipoprotein-associated phospholipase A2 during the hyperacute stage of ischemic and hemorrhagic strokes. J Stroke Cerebrovasc Dis 23: 277-282, 2014

16. Tsimikas S, Willeit J, Knoflach M, Mayr M, Egger G, Notdurfter M, Witztum JL, Wiedermann CJ, Xu Q and Kiechl S: Lipoprotein-associated phospholipase A2 activity, ferritin levels, metabolic syndrome and 10-year cardiovascular and non-cardiovascular mortality: Results from the Bruneck study. Eur Heart J 30: 107-115, 2009.

17. Wang Q, Hao Y, Mo X, Wang L, Lu X, Huang J, Cao J, Li H and Gu D: PLA2G7 gene polymorphisms and coronary heart disease risk: A meta-analysis. Thromb Res 126: 498-503, 2010.

18. Zalewski A and Macphee C: Role of lipoprotein-associated phospholipase A2 in atherosclerosis: Biology, epidemiology and possible therapeutic target. Arterioscler Thromb Vasc Biol 25: 923-931, 2005 .

19. Macphee $\mathrm{CH}$, Nelson JJ and Zalewski A: Lipoprotein-associated phospholipase A2 as a target of therapy. Curr Opin Lipidol 16: 442-446, 2005.

20. Vittos O, Toana B, Vittos A and Moldoveanu E: Lipoprotein-associated phospholipase A2 (Lp-PLA2): A review of its role and significance as a cardiovascular biomarker. Biomarkers 17: 289-302, 2012. 